Research Article

\title{
Iterative Method for a System of Nonlinear Fredholm Integral Equations
}

\author{
Keyan Wang $\mathbb{D}^{1}$ and Qisheng Wang $\mathbb{D}^{2}$ \\ ${ }^{1}$ School of Mathematics and Statistics, Hengyang Normal University, Hengyang 421008, Hunan, China \\ ${ }^{2}$ School of Mathematics and Computational Science, Wuyi University, Jiangmen 529020, Guangdong, China
}

Correspondence should be addressed to Qisheng Wang; 282228006@qq.com

Received 15 July 2020; Accepted 14 December 2020; Published 8 January 2021

Academic Editor: Mariusz Michta

Copyright (C) 2021 Keyan Wang and Qisheng Wang. This is an open access article distributed under the Creative Commons Attribution License, which permits unrestricted use, distribution, and reproduction in any medium, provided the original work is properly cited.

\begin{abstract}
In this paper, the iteration method is proposed to solve a class of system of Fredholm-type nonlinear integral equations. First, the existence and uniqueness of solution are theoretically proven by the fixed-point theorem. Second, the approximation solution method is given by using the appropriate integration rule. The error analysis for the approximated solution with the exact solution is discussed for infinity-norm, and the rates of convergence are obtained. Furthermore, an iteration algorithm is constructed, and the convergence of the proposed numerical method is rigorously derived. Finally, some numerical examples are given to illustrate the theoretical results.
\end{abstract}

\section{Introduction}

In this paper, a system of nonlinear Fredholm integral equations can be written as follows:

$$
\begin{gathered}
u_{p}(x)=f_{p}(x)+\int_{a}^{b} \sum_{q=1}^{n} \lambda_{p q} k_{p q}(x, y) \phi_{p q}\left(y, u_{q}(y)\right) \mathrm{d} y \\
p=1,2, \ldots, n,
\end{gathered}
$$

where $x \in[a, b], \lambda_{p q} \in \mathbb{R}, f_{p}, k_{p q}$, and $\phi_{p q}, p, q=1,2, \ldots, n$, are known functions, and $u_{p}(x)$ are unknown functions.

Integral equations appear in numerous fields of physics, chemistry, biology, engineering, etc. They can be employed to model many phenomena and processes occurring in the real world so that these equations have received a great deal of attention and research. In the past, many approximate analytical methods have been applied to solve Fredholm integral problems such as spectral collocation method [1], Taylor polynomial method [2, 3], Nyström method [4], and iterative method [5-7]. Therefore, the development of an effective computational method for investigating (1) has practical significance.

The Nyström method is usually regarded as an efficient discretization technique for solving the linear and nonlinear integral equations, which was introduced by Nyström in 1930 and has been developed for the weakly singular and singular integral equations in [8-10] and references therein. From then on, the method was further investigated and applied to other problems by many authors. For instance, Han and Wang [4] have obtained the asymptotic error expansions for numerical solutions of two-dimensional nonlinear Fredholm integral equations by using the Nyström method. In [11], the Nyström method was introduced for a class of integral equations on the real line with applications to scattering by diffraction gratings and rough surfaces. Recently, the Nyström method also has been applied to the Urysohn integral equations [12], Volterra integro-differential equations [13], Mellin convolution equations [14], and multifrequency oscillatory systems [15]. Influenced by the work mentioned above, the goal of this work is to develop the Nyström method for problem (1) and provide rigorous error analysis for the method. 
For Fredholm integral equations, the iterated method [5-7] is used to accelerate the approximation. Comparing with [6], under some suitable conditions for $\lambda_{p q}$ and $k_{p q}$, we need not change and narrow the interval of integral equations. To the best of our knowledge, there is no iterated method convergence analysis for problem (1) in the literature that is combined with the Nyström method. In this paper, we develop the method and the corresponding convergence analysis which partly fill this gap. Our purpose is two fold. First of all, we propose the Nyström method to approximate (1). Secondly, in order to accelerate the approximation, we use the iterated algorithm to solve the Nyström method equations.
The organization of this paper is as follows. In Section 2, the existence and uniqueness of solution for (1) is proven, the Nyström method is stated, and the error estimates are derived. In Section 3, an iterated algorithm is defined and the convergence result is deduced. In Section 4, we present two numerical examples which show the efficiency of the proposed method. In Section 5, some conclusions are presented.

\section{The Nyström Method and Error Analysis}

For simplicity, let us consider the system of nonlinear integral equation (1) with $n=2$ :

$$
\left\{\begin{array}{l}
u_{1}(x)=f_{1}(x)+\int_{a}^{b}\left(\lambda_{11} k_{11}(x, y) \phi_{11}\left(y, u_{1}(y)\right)+\lambda_{12} k_{12}(x, y) \phi_{12}\left(y, u_{2}(y)\right)\right) \mathrm{d} y \\
u_{2}(x)=f_{2}(x)+\int_{a}^{b}\left(\lambda_{21} k_{21}(x, y) \phi_{21}\left(y, u_{1}(y)\right)+\lambda_{22} k_{22}(x, y) \phi_{22}\left(y, u_{2}(y)\right)\right) \mathrm{d} y
\end{array}\right.
$$

2.1. The Existence and Uniqueness of Solution. In this section, we discuss the existence and uniqueness of solution for (1). For convenience, we rewrite (2) in the form

$$
\left(\begin{array}{l}
u_{1}(x) \\
u_{2}(x)
\end{array}\right)=\left(\begin{array}{l}
f_{1}(x) \\
f_{2}(x)
\end{array}\right)+\int_{a}^{b}\left[\begin{array}{l}
\lambda_{11} k_{11}(x, y) \phi_{11}\left(y, u_{1}(y)\right)+\lambda_{12} k_{12}(x, y) \phi_{12}\left(y, u_{2}(y)\right) \\
\lambda_{21} k_{21}(x, y) \phi_{21}\left(y, u_{1}(y)\right)+\lambda_{22} k_{22}(x, y) \phi_{22}\left(y, u_{2}(y)\right)
\end{array}\right] \mathrm{d} y
$$

Using the well-known Banach fixed-point theorem, one can easily prove that the solution of (2) exists and is unique in the interval $[a, b]$. The uniqueness of a solution $\left[u_{1} ; u_{2}\right]$ to (2) is provided in the following lemma.
Lemma 1. Assume that $k_{p q} \in C([a, b] \times[a, b])$ and $\phi_{p q} \in C^{1}\left([a, b] \times\left[u_{q, \min }, u_{q, \max }\right]\right), p, q=1,2$. If the following condition is satisfied,

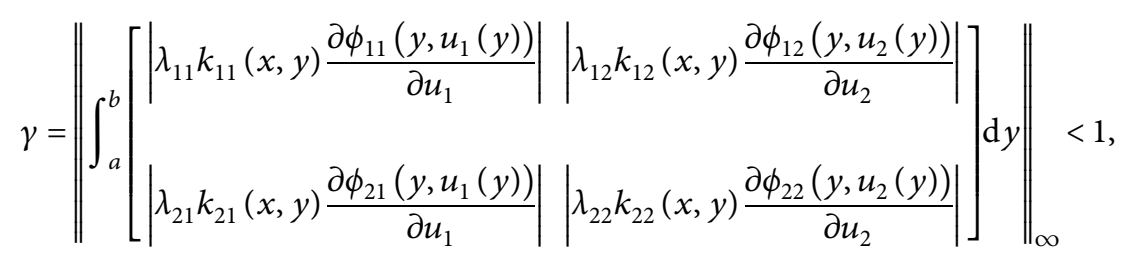

then (2) exists as an unique solution.

Proof. In a suitable Banach space, we define the operator:

$$
T\left(\begin{array}{c}
u_{1} \\
u_{2}
\end{array}\right)=\left(\begin{array}{c}
f_{1} \\
f_{2}
\end{array}\right)+\int_{a}^{b}\left[\begin{array}{c}
\lambda_{11} k_{11} \phi_{11}+\lambda_{12} k_{12} \phi_{12} \\
\lambda_{21} k_{21} \phi_{21}+\lambda_{22} k_{22} \phi_{22}
\end{array}\right] \mathrm{d} y
$$

$$
T\left(\begin{array}{l}
u_{1,1} \\
u_{2,1}
\end{array}\right)-T\left(\begin{array}{l}
u_{1,2} \\
u_{2,2}
\end{array}\right)=\int_{a}^{b}\left[\begin{array}{l}
\lambda_{11} k_{11}\left(\phi_{11}\left(y, u_{1,1}\right)-\phi_{11}\left(y, u_{1,2}\right)\right)+\lambda_{12} k_{12}\left(\phi_{12}\left(y, u_{2,1}\right)-\phi_{12}\left(y, u_{2,2}\right)\right) \\
\lambda_{21} k_{21}\left(\phi_{21}\left(y, u_{1,1}\right)-\phi_{21}\left(y, u_{1,2}\right)\right)+\lambda_{22} k_{22}\left(\phi_{22}\left(y, u_{2,1}\right)-\phi_{22}\left(y, u_{2,2}\right)\right)
\end{array}\right] \mathrm{d} y
$$


Since

$$
\phi_{p q}\left(y, u_{q, 1}\right)-\phi_{p q}\left(y, u_{q, 2}\right)=\left.\frac{\partial \phi_{p q}}{\partial u_{q}}\right|_{u_{q}=\xi_{p q}(y)}\left(u_{q, 1}-u_{q, 2}\right),
$$

where $\xi_{p q}, p, q=1,2$, are some values between $u_{q, 1}$ and $u_{q, 2}$. Then,

$$
T\left(\begin{array}{c}
u_{1,1} \\
u_{2,1}
\end{array}\right)-T\left(\begin{array}{c}
u_{1,2} \\
u_{2,2}
\end{array}\right)=\int_{a}^{b}\left[\begin{array}{ll}
\left.\lambda_{11} k_{11} \frac{\partial \phi_{11}}{\partial u_{1}}\right|_{u_{1}=\xi_{11}} & \left.\lambda_{12} k_{12} \frac{\partial \phi_{12}}{\partial u_{2}}\right|_{u_{2}=\xi_{12}} \\
\left.\lambda_{21} k_{21} \frac{\partial \phi_{21}}{\partial u_{1}}\right|_{u_{1}=\xi_{21}} & \left.\lambda_{22} k_{22} \frac{\partial \phi_{22}}{\partial u_{2}}\right|_{u_{2}=\xi_{22}}
\end{array}\right]\left[\begin{array}{l}
u_{1,1}-u_{1,2} \\
u_{2,1}-u_{2,2}
\end{array}\right] \mathrm{d} y
$$

and therefore

$$
\begin{aligned}
\left\|T\left(\begin{array}{c}
u_{1,1} \\
u_{2,1}
\end{array}\right)-T\left(\begin{array}{c}
u_{1,2} \\
u_{2,2}
\end{array}\right)\right\|_{\infty} & \leq\left\|\int_{a}^{b}\left[\begin{array}{ll}
\lambda_{11} k_{11} \frac{\partial \phi_{11}}{\partial u_{1}} & \lambda_{12} k_{12} \frac{\partial \phi_{12}}{\partial u_{2}} \\
\lambda_{21} k_{21} \frac{\partial \phi_{21}}{\partial u_{1}} & \lambda_{22} k_{22} \frac{\partial \phi_{22}}{\partial u_{2}}
\end{array}\right] \mathrm{d} y\right\|\left\|_{\infty}\right\| u_{1,1}-u_{1,2}\left\|_{2,2}\right\|_{\infty} \\
& \leq \gamma\left\|\left(\begin{array}{c}
u_{1,1} \\
u_{2,1}
\end{array}\right)-\left(\begin{array}{c}
u_{1,2} \\
u_{2,2}
\end{array}\right)\right\|_{\infty} .
\end{aligned}
$$

Note that $0 \leq \gamma<1$, so $T$ is a contraction mapping; then, there exists a unique fixed point $\left[u_{1} ; u_{2}\right]$ such that $T\left(\begin{array}{l}u_{1} \\ u_{2}\end{array}\right)=\left(\begin{array}{l}u_{1} \\ u_{2}\end{array}\right)$.

The existence and uniqueness of a solution $\mathbf{U}(x)=\left[u_{1}(x), u_{2}(x), \ldots, u_{n}(x)\right]^{T}$ to $(1)$ is shown in the following theorem.

Theorem 1. A solution $\mathrm{U}(x)$ to (1) exists and is unique.

Proof. Uniqueness follows directly from the proof of the previous lemma.
2.2. Description of the Method. Consider an interval $[a, b]$ in which we select $n+1$ equidistant nodes $a=x_{0}<x_{1}<\cdots<x_{n}=b, \quad$ where $\quad h=x_{i}-x_{i-1}$, $i=1,2, \ldots, n$. Let

$$
\int_{a}^{b} \psi(x, y) \mathrm{d} y=\sum_{j=0}^{n} \omega_{j} \psi\left(x, x_{j}\right)+R_{n}, \quad a \leq x \leq b,
$$

be a numerical integration formula, where $\omega_{j}$ and $x_{j}=a+j h, h=(b-a) / n$ and $j=0,1, \ldots, n$, are the coefficients and nodes of quadrature and $R_{n}$ is the residual error for integration. Using (10) in (2) and neglecting the error $R_{n}$, we have

$$
\left\{\begin{array}{l}
u_{1}(x)=f_{1}(x)+\sum_{j=0}^{n} \omega_{j}\left(\lambda_{11} k_{11}\left(x, x_{j}\right) \phi_{11}\left(x_{j}, u_{1}\left(x_{j}\right)\right)+\lambda_{12} k_{12}\left(x, x_{j}\right) \phi_{12}\left(x_{j}, u_{2}\left(x_{j}\right)\right)\right), \\
u_{2}(x)=f_{2}(x)+\sum_{j=0}^{n} \omega_{j}\left(\lambda_{21} k_{21}\left(x, x_{j}\right) \phi_{21}\left(x_{j}, u_{1}\left(x_{j}\right)\right)+\lambda_{22} k_{22}\left(x, x_{j}\right) \phi_{22}\left(x_{j}, u_{2}\left(x_{j}\right)\right)\right) .
\end{array}\right.
$$


In the following, using the collocation points $x=x_{i}$, $i=0,1, \ldots, n$, we rewrite (11) as follows:

$$
\left\{\begin{array}{l}
u_{1 i}=f_{1 i}+\sum_{j=0}^{n} \omega_{j}\left(\lambda_{11} k_{11}^{i j} \phi_{11}^{j}\left(u_{1 j}\right)+\lambda_{12} k_{12}^{i j} \phi_{12}^{j}\left(u_{2 j}\right)\right) \\
u_{2 i}=f_{2 i}+\sum_{j=0}^{n} \omega_{j}\left(\lambda_{21} k_{21}^{i j} \phi_{21}^{j}\left(u_{1 j}\right)+\lambda_{22} k_{22}^{i j} \phi_{22}^{j}\left(u_{2 j}\right)\right)
\end{array}\right.
$$

where $u_{p i}=u_{p}\left(x_{i}\right), f_{p i}=f_{p}\left(x_{i}\right), k_{p q}^{i j}=k_{p q}\left(x_{i}, x_{j}\right)$, and $\phi_{p q}^{j}\left(u_{p j}\right)=\phi_{p q}\left(x_{j}, u_{q}\left(x_{j}\right)\right), p, q=1,2$ and $i, j=0,1, \ldots, n$. Let $u_{p i}=u_{p i}^{*}, p=1,2$ and $i=0,1, \ldots, n$, be the solutions of
(12). Now, we substitute these solutions into (11), and there is

$$
\left\{\begin{array}{l}
\bar{u}_{1}\left(x_{i}\right)=f_{1}(x)+\sum_{j=0}^{n} w_{j}\left(\lambda_{11} k_{11}^{i j}\left(x, x_{j}\right) \phi_{11}^{j}\left(x_{j}, u_{1 j}^{*}\right)+\lambda_{12} k_{12}^{i j}\left(x, x_{j}\right) \phi_{12}^{j}\left(x_{j}, u_{2 j}^{*}\right)\right), \\
\bar{u}_{2}\left(x_{i}\right)=f_{1}(x)+\sum_{j=0}^{n} w_{j}\left(\lambda_{21} k_{21}^{i j}\left(x, x_{j}\right) \phi_{21}^{j}\left(x_{j}, u_{1 j}^{*}\right)+\lambda_{22} k_{22}^{i j}\left(x, x_{j}\right) \phi_{22}^{j}\left(x_{j}, u_{2 j}^{*}\right)\right) .
\end{array}\right.
$$

By (13), we can obtain the Nyström approximation solution $\left[\bar{u}_{1}(x) ; \bar{u}_{2}(x)\right]$ of $(2)$.

\section{Remark 1}

(I) For $i=0,1, \ldots, n$, the solution $\left[\bar{u}_{1}\left(x_{i}\right) ; \bar{u}_{2}\left(x_{i}\right)\right]$ of (13) satisfies

$$
\left\{\begin{array}{l}
\bar{u}_{1}\left(x_{i}\right)=u_{1 i}^{*} \\
\bar{u}_{2}\left(x_{i}\right)=u_{2 i}^{*}
\end{array}\right.
$$

(II) It is seen that when the composite trapezoidal and Simpson rules are used to solve (13), the given method is the special case of the Nyström-type method.

2.3. Error Analysis. In this section, the error estimation of (13) in the infinite norm sense and the results of convergence analysis are given. Let

$$
M_{p q}=\left\|\sum_{j=1}^{n} \omega_{j} \lambda_{p q} k_{p q} \frac{\partial \phi_{p q}}{\partial u_{q}}\right\|_{\infty}
$$

for $p, q=1,2$. Now, we prove the following lemma.

Lemma 2. Let $\left[u_{1}(x) ; u_{2}(x)\right]$ and $\left[\bar{u}_{1}(x) ; \bar{u}_{2}(x)\right]$ be the solutions of (2) and (13), respectively. Assume that $k_{p q} \in C([a, b] \times[a, b]) \quad$ and $\quad \phi_{p q} \in C^{1}\left([a, b] \times\left[u_{q, \min }\right.\right.$, $\left.\left.u_{q, \max }\right]\right), p, q=1,2$. If the following condition is satisfied

$$
\beta=\sum_{p, q=1}^{2} M_{p q}<\frac{1}{2}
$$

then we have

$$
\left\|u_{1}-\bar{u}_{1}\right\|_{\infty}+\left\|u_{2}-\bar{u}_{2}\right\|_{\infty} \leq \frac{\left\|R_{1}^{(n)}\right\|_{\infty}+\left\|R_{2}^{(n)}\right\|_{\infty}}{1-2 \beta} .
$$

Proof. Substituting (10) into (2) yields

$$
\left\{\begin{array}{l}
u_{1}(x)=f_{1}(x)+\sum_{j=0}^{n} \omega_{j}\left(\lambda_{11} k_{11}\left(x, x_{j}\right) \phi_{11}\left(x_{j}, u_{1}\left(x_{j}\right)\right)+\lambda_{12} k_{12}\left(x, x_{j}\right) \phi_{12}\left(x_{j}, u_{2}\left(x_{j}\right)\right)\right)+R_{1}^{(n)}, \\
u_{2}(x)=f_{2}(x)+\sum_{j=0}^{n} \omega_{j}\left(\lambda_{21} k_{21}\left(x, x_{j}\right) \phi_{21}\left(x_{j}, u_{1}\left(x_{j}\right)\right)+\lambda_{22} k_{22}\left(x, x_{j}\right) \phi_{22}\left(x_{j}, u_{2}\left(x_{j}\right)\right)\right)+R_{2}^{(n)}
\end{array}\right.
$$


It follows from (13) and (18) that

$$
\begin{aligned}
u_{1}(x)-\bar{u}_{1}(x)= & \sum_{j=0}^{n} \omega_{j}\left[\lambda_{11} k_{11}\left(x, x_{j}\right)\left(\phi_{11}\left(x_{j}, u_{1}\left(x_{j}\right)\right)-\phi_{11}\left(x_{j}, \bar{u}_{1}\left(x_{j}\right)\right)\right)\right] \\
& \left.+\lambda_{12} k_{12}\left(x, x_{j}\right)\left(\phi_{12}\left(x_{j}, u_{2}\left(x_{j}\right)\right)-\phi_{12}\left(x_{j}, \bar{u}_{2}\left(x_{j}\right)\right)\right)\right]+R_{1}^{(n)} \\
= & \sum_{j=0}^{n} \omega_{j}\left[\left.\lambda_{11} k_{11}\left(x, x_{j}\right) \frac{\partial \phi_{11}\left(x_{j}, u_{1}\right)}{\partial u_{1}}\right|_{u_{1}=\xi_{11}\left(x_{j}\right)}\left(u_{1}\left(x_{j}\right)-\bar{u}_{1}\left(x_{j}\right)\right)\right] \\
& \left.+\left.\lambda_{12} k_{12}\left(x, x_{j}\right) \frac{\partial \phi_{12}\left(x_{j}, u_{2}\right)}{\partial u_{2}}\right|_{u_{2}=\xi_{12}\left(x_{j}\right)}\left(u_{2}\left(x_{j}\right)-\bar{u}_{2}\left(x_{j}\right)\right)\right]+R_{1}^{(n)},
\end{aligned}
$$

and therefore,

$$
\begin{aligned}
\left\|u_{1}-\bar{u}_{1}\right\|_{\infty} & \leq M_{11} \max _{0 \leq j \leq n}\left|u_{1}\left(x_{j}\right)-\bar{u}_{1}\left(x_{j}\right)\right|+M_{12} \max _{0 \leq j \leq n}\left|u_{2}\left(x_{j}\right)-\bar{u}_{2}\left(x_{j}\right)\right|+\left\|R_{1}^{(n)}\right\|_{\infty} \\
& \leq M_{11}\left\|u_{1}-\bar{u}_{1}\right\|_{\infty}+M_{12}\left\|u_{2}-\bar{u}_{2}\right\|_{\infty}+\left\|R_{1}^{(n)}\right\|_{\infty} \\
& \leq\left(M_{11}+M_{12}\right)\left(\left\|u_{1}-\bar{u}_{1}\right\|_{\infty}+\left\|u_{2}-\bar{u}_{2}\right\|_{\infty}\right)+\left\|R_{1}^{(n)}\right\|_{\infty} \\
& \leq \beta\left(\left\|u_{1}-\bar{u}_{1}\right\|_{\infty}+\left\|u_{2}-\bar{u}_{2}\right\|_{\infty}\right)+\left\|R_{1}^{(n)}\right\|_{\infty},
\end{aligned}
$$

where $\beta=\sum_{p, q=1}^{2} M_{p q}$ and $M_{p q}(p, q=1,2)$ are as described in (15). Using the same argument as estimate (20), we deduce that there holds

$$
\left\|u_{2}-\bar{u}_{2}\right\|_{\infty} \leq \beta\left(\left\|u_{1}-\bar{u}_{1}\right\|_{\infty}+\left\|u_{2}-\bar{u}_{2}\right\|_{\infty}\right)+\left\|R_{2}^{(n)}\right\|_{\infty} .
$$

By (20) and (21), we have

$$
\begin{gathered}
\left\|u_{1}-\bar{u}_{1}\right\|_{\infty}+\left\|u_{2}-\bar{u}_{2}\right\|_{\infty} \leq 2 \beta\left(\left\|u_{1}-\bar{u}_{1}\right\|_{\infty}+\left\|u_{2}-\bar{u}_{2}\right\|_{\infty}\right) \\
+\left\|R_{1}^{(n)}\right\|_{\infty}+\left\|R_{2}^{(n)}\right\|_{\infty} .
\end{gathered}
$$

Let the value $\beta<(1 / 2)$, and we can rewrite (22) as

$$
\left\|u_{1}-\bar{u}_{1}\right\|_{\infty}+\left\|u_{2}-\bar{u}_{2}\right\|_{\infty} \leq \frac{\left\|R_{1}^{(n)}\right\|_{\infty}+\left\|R_{2}^{(n)}\right\|_{\infty}}{1-2 \beta},
$$

which completes the proof.

From the above analysis and [16], we can also obtain the following results.

Corollary 1. Assume that $\left[u_{1}(x) ; u_{2}(x)\right]$ and $\left[\bar{u}_{1}(x) ; \bar{u}_{2}(x)\right]$ are as described in Lemma 2, $k_{p q} \in C^{2}([a, b] \times[a, b]), \quad$ and $\quad \phi_{p q} \in C^{2}([a, b] \times$ $\left.\left[u_{q, \min }, u_{q, \max }\right]\right), p, q=1,2$. If (16) is satisfied and using the composite trapezoidal integration formula in (13), then we have

$$
\left\|u_{1}-\bar{u}_{1}\right\|_{\infty}+\left\|u_{2}-\bar{u}_{2}\right\|_{\infty}=\mathcal{O}\left(h^{2}\right) .
$$

Corollary 2. Assume that $k_{p q} \in C^{4}([a, b] \times[a, b])$ and $\phi_{p q} \in C^{4}\left([a, b] \times\left[u_{q, \min }, u_{q, \max }\right]\right), \quad p, q=1,2$. Using the Simpson integration formula in (13), thus

$$
\left\|u_{1}-\bar{u}_{1}\right\|_{\infty}+\left\|u_{2}-\bar{u}_{2}\right\|_{\infty}=\mathcal{O}\left(h^{4}\right) .
$$

From (17), (24), and (25), when the value of $n \longrightarrow \infty$, we obtain the following corollary.

Corollary 3. $\left\|u_{1}-\bar{u}_{1}\right\|_{\infty}+\left\|u_{2}-\bar{u}_{2}\right\|_{\infty}$ vanishes when $h$ tends to zero.

In the same manner as it has been carried out in the proofs of Lemma 2 and Corollary 3, we can derive the estimate of $\sum_{p=1}^{n}\left\|u_{p}-\bar{u}_{p}\right\|_{\infty}$.

Theorem 2. Let $u_{p}(x), p=1,2, \ldots, n$, be the solutions of $(1)$. Assume that $k_{p q} \in C([a, b] \times[a, b])$ and $\phi_{p q} \in C^{1}\left([a, b] \times\left[u_{q, \min }, u_{q, \max }\right]\right), p, q=1,2, \ldots, n$. If the following condition is satisfied $\beta=\sum_{p, q=1}^{n} M_{p q}<(1 / n)$, then we have
(I) $\sum_{p=1}^{n}\left\|u_{p}-\bar{u}_{p}\right\|_{\infty} \leq\left(\left(\sum_{p=1}^{n}\left\|R_{p}^{(n)}\right\|_{\infty}\right) /(1-n \beta)\right)$
(II) $\sum_{p=1}^{n}\left\|u_{p}-\bar{u}_{p}\right\|_{\infty}$ vanishes when $h \longrightarrow 0$ 


\section{The Iterative Algorithm and Convergence Analysis}

In this section, we will consider a numerical method to solve the nonlinear system (13) by using Newton's method. First, we define two vector sequences $\left\{\bar{u}_{1}^{(k)}\right\}$ and $\left\{\bar{u}_{2}^{(k)}\right\}$ and apply the iterative formula; thus, (13) becomes

$$
\left\{\begin{array}{l}
\bar{u}_{1}^{(k+1)}\left(x_{i}\right)=f_{1}\left(x_{i}\right)+\sum_{j=0}^{n} \omega_{j}\left(\lambda_{11} k_{11}^{i j} \phi_{11}\left(x_{j}, \bar{u}_{1}^{(k)}\left(x_{j}\right)\right)+\lambda_{12} k_{12}^{i j} \phi_{12}\left(x_{j}, \bar{u}_{2}^{(k)}\left(x_{j}\right)\right)\right), \\
\bar{u}_{2}^{(k+1)}\left(x_{i}\right)=f_{2}\left(x_{i}\right)+\sum_{j=0}^{n} \omega_{j}\left(\lambda_{21} k_{21}^{i j} \phi_{21}\left(x_{j}, \bar{u}_{1}^{(k)}\left(x_{j}\right)\right)+\lambda_{22} k_{22}^{i j} \phi_{22}\left(x_{j}, \bar{u}_{2}^{(k)}\left(x_{j}\right)\right)\right),
\end{array}\right.
$$

where $i=0,1, \ldots, n$ and $k=0,1, \ldots$

In order to solve (26), we construct the iteration algorithm (Algorithm 1).

Lemma 3. Considering assumptions of Lemma 2, the produced sequences $\left\{\bar{u}_{1}^{(k)}\right\}$ and $\left\{\bar{u}_{2}^{(k)}\right\}$ from the iteration process
(26) tend to $\left[\bar{u}_{1 i} ; \bar{u}_{2 i}\right]$ of (13), for any arbitrary initial vector $\left[\bar{u}_{1}^{(0)} ; \bar{u}_{2}^{(0)}\right]$.

Proof. By (12) and (26), we obtain

$$
\left\{\begin{array}{l}
\bar{u}_{1 i}^{(k+1)}-\bar{u}_{1 i}=\sum_{j=0}^{n} \omega_{j}\left[\lambda_{11} k_{11}^{i j}\left(\phi_{11}\left(x_{j}, \bar{u}_{1 j}^{(k)}\right)-\phi_{11}\left(x_{j}, \bar{u}_{1 j}\right)\right)+\lambda_{12} k_{12}^{i j}\left(\phi_{12}\left(x_{j}, \bar{u}_{2 j}^{(k)}\right)-\phi_{12}\left(x_{j}, \bar{u}_{2 j}\right)\right)\right], \\
\bar{u}_{2 i}^{(k+1)}-\bar{u}_{2 i}=\sum_{j=0}^{n} \omega_{j}\left[\lambda_{21} k_{21}^{i j}\left(\phi_{21}\left(x_{j}, \bar{u}_{1 j}^{(k)}\right)-\phi_{21}\left(x_{j}, \bar{u}_{1 j}\right)\right)+\lambda_{22} k_{22}^{i j}\left(\phi_{22}\left(x_{j}, \bar{u}_{2 j}^{(k)}\right)-\phi_{22}\left(x_{j}, \bar{u}_{2 j}\right)\right)\right],
\end{array}\right.
$$

where $i=0,1, \ldots, n$. According to the condition of Lemma

2 , we have

$$
\left|\sum_{j=0}^{n} \omega_{j} \lambda_{p q} k_{p q}^{i j}\left(\phi_{p q}\left(x_{j}, \bar{u}_{q j}^{(k)}\right)-\phi_{p q}\left(x_{j}, \bar{u}_{q j}\right)\right)\right|=\left|\sum_{j=0}^{n} \omega_{j} \lambda_{p q} k_{p q}^{i j} \frac{\partial \phi_{p q}}{\partial u_{q}}\left(x_{j}, \xi_{p q}^{j(k)}\right)\left(\bar{u}_{q j}^{(k)}-\bar{u}_{q j}\right)\right| \leq M_{p q}\left|\bar{u}_{q j}^{(k)}-\bar{u}_{q j}\right|,
$$

where $p, q=1,2$ and $\xi_{p q}^{j(k)}, j=0,1, \ldots, n$, are some real numbers between $\bar{u}_{q j}^{(k)}$ and $\bar{u}_{q j}$. It follows from (27) and (28) that

$$
\begin{aligned}
&\left\|\bar{u}_{1 i}^{(k+1)}-\bar{u}_{1 i}\right\|_{\infty} \leq \beta\left(\left\|\bar{u}_{1 i}^{(k)}-\bar{u}_{1 i}\right\|_{\infty}+\left\|\bar{u}_{2 i}^{(k)}-\bar{u}_{2 i}\right\|_{\infty}\right) \\
& \leq 2 \beta^{2}\left(\left\|\bar{u}_{1 i}^{(k-1)}-\bar{u}_{1 i}\right\|_{\infty}+\left\|\bar{u}_{2 i}^{(k-1)}-\bar{u}_{2 i}\right\|_{\infty}\right) \\
& \leq \cdots \leq 2^{k} \beta^{k+1}\left(\left\|\bar{u}_{1 i}^{(0)}-\bar{u}_{1 i}\right\|_{\infty}+\left\|\bar{u}_{1 i}^{(0)}-\bar{u}_{2 i}\right\|_{\infty}\right), \\
&\left\|\bar{u}_{2 i}^{(k+1)}-\bar{u}_{2 i}\right\|_{\infty} \leq 2^{k} \beta^{k+1}\left(\left\|\bar{u}_{1 i}^{(0)}-\bar{u}_{1 i}\right\|_{\infty}+\left\|\bar{u}_{2 i}^{(0)}-\bar{u}_{2 i}\right\|_{\infty}\right) .
\end{aligned}
$$


Combining estimates (29) and (30), we find that

$$
\left\|\bar{u}_{1 i}^{(k+1)}-\bar{u}_{1 i}\right\|_{\infty}+\left\|\bar{u}_{2 i}^{(k+1)}-\bar{u}_{2 i}\right\|_{\infty} \leq(2 \beta)^{k+1}\left(\left\|\bar{u}_{1 i}^{(0)}-\bar{u}_{1 i}\right\|_{\infty}+\left\|\bar{u}_{2 i}^{(0)}-\bar{u}_{2 i}\right\|_{\infty}\right)
$$

for each $k=0,1, \ldots$. Since $\beta<(1 / 2)$, then $k \longrightarrow \infty$ implies that

$$
\lim _{k \rightarrow \infty}\left(\left\|\bar{u}_{1 i}^{(k+1)}-\bar{u}_{1 i}\right\|_{\infty}+\left\|\bar{u}_{2 i}^{(k+1)}-\bar{u}_{2 i}\right\|_{\infty}\right)=0 .
$$

Thus, the proof is completed.

From the above analysis, we can also extend the result of the iterative method to (1).

Theorem 3. Assume that the sequences $\left\{\bar{u}_{p}^{(k)}\right\}$ and $\left\{\bar{u}_{p}\right\}$, $p=1,2, \ldots, n$, are the iterative and Nyström approximation solutions, respectively. Then, we have $\lim _{k \rightarrow \infty} \sum_{p=1}^{n} \| \bar{u}_{p}^{(k)}$ $-\bar{u}_{p} \|_{\infty}=0$, where $\bar{u}_{p}^{(k)}=\left(\bar{u}_{p 0}^{(k)}, \bar{u}_{p 1}^{(k)}, \ldots, \bar{u}_{p n}^{(k)}\right)^{p=1}$ and $\bar{u}_{p}=\left(\bar{u}_{p 0}, \bar{u}_{p 1}, \ldots, \bar{u}_{p n}\right)$.

\section{Numerical Examples}

In this section, we present two numerical examples to verify the above algorithm and the theoretical estimates obtained in the previous sections. In the numerical examples reported below, we define a discrete error function $E=\left\|u_{p}-\bar{u}_{p}\right\|_{\infty}=\max _{a \leq x_{i} \leq b}\left|u_{p}\left(x_{i}\right)-\bar{u}_{p}^{k}\left(x_{i}\right)\right|, \quad$ where $x_{i}=a+(b-a / n) i, i=0,1, \ldots, n$. In addition, the convergence rate is defined as $\log _{2}\left(E_{2 h} / E_{h}\right)$, and we select $\varepsilon=1 e-$ 10 as the tolerance error of the iteration in real calculations. All the computations are carried out in Matlab 2012b.

Example 1. Consider the following problem:

$$
\left\{\begin{array}{l}
u_{1}(x)=f_{1}(x)+\frac{1}{240} \int_{0}^{1} \frac{1}{300+2 x}\left(1+\left(\frac{\cos y}{5}\right)^{4}\right) u_{1}^{4}(y) \mathrm{d} y+\frac{1}{50} \int_{0}^{1} \frac{1}{e^{x}} \cos y \sin \left(u_{2}(y)\right) \mathrm{d} y, \\
u_{2}(x)=f_{2}(x)+\frac{1}{100} \int_{0}^{1} \frac{x e^{y}}{40+x^{2}}\left(y+\left(\frac{u_{1}(y)}{4}\right)^{4}\right) \mathrm{d} y+\frac{1}{20} \int_{0}^{1} \frac{1}{10+x} \sin y \cos \left(u_{2}(y)\right) \mathrm{d} y,
\end{array}\right.
$$

where $0 \leq x \leq 1$ and $f_{1}(x)$ and $f_{2}(x)$ are suitably chosen such that the exact solution $\left[u_{1}(x) ; u_{2}(x)\right]=[\sin x ; 2 x]$. Conditions (4) and (16) hold; the solution of (33) will be approximated by the Nyström method, and the resulting nonlinear algebraic systems are solved by the Newton iteration method.

The numerical results of the iteration method with $n=$ $\{1,2,4,8,16\}$ are demonstrated in Tables 1 and 2. From Tables 1 and 2, we see that the relative errors decrease as the mesh becomes fine, and the numerical results calculated by the composite Simpson rule have more accuracy. Moreover, we also observe from Tables 1 and 2 that the convergence rates are two-order and four-order for the composite trapezoidal and Simpson rules, respectively. These values also indicate agreement with predicted convergence rates. Table 3 represents the error estimates using the methods of $[17,18]$. From Tables $1-3$, compared with the methods of $[17,18]$, our method is very well in numerical solution of $(1)$.

Example 2. Consider the one-dimensional nonlinear system of Fredholm integral equations:

$$
\left\{\begin{array}{l}
u_{1}(x)=f_{1}(x)+\frac{1}{160} \int_{0}^{1}\left(e^{x}-1-\sin y\right) \cos \left(u_{1}(y)\right) \mathrm{d} y+\frac{1}{400} \int_{0}^{1} \frac{y e^{y^{2}}}{\left(80+x^{2}\right)} e^{u_{2}(y)} \mathrm{d} y+\frac{1}{50} \int_{0}^{1} \sin x \sin y\left(1-\log \left(u_{3}(y)\right)\right) \mathrm{d} y, \\
u_{2}(x)=f_{2}(x)+\frac{1}{160} \int_{0}^{1}\left(e^{x}-\frac{x^{2}}{2}-\cos y\right) e^{u_{1}(y)} \mathrm{d} y+\frac{1}{240} \int_{0}^{1} \frac{y \cos x \sin \left(y^{2}\right)}{10+2 x} \cos \left(u_{2}(y)\right) \mathrm{d} y+\frac{1}{50} \int_{0}^{1} \cos x \cos y \log \left(u_{3}(y)\right) \mathrm{d} y \\
=f_{3}(x)+\frac{1}{160} \int_{0}^{1}\left(\frac{e^{y}}{5}-\frac{1}{1+x}\right) \sin \left(u_{1}(y)\right) \mathrm{d} y+\frac{1}{100} \int_{0}^{1}\left(\frac{x}{4}+\frac{y}{6}\right)^{4}\left(1-\left(\frac{u_{2}(y)}{5}\right)^{5}\right) \mathrm{d} y+\frac{1}{200} \int_{0}^{1} \sin x \cos y\left(\frac{y}{6}+\log \left(u_{3}(y)\right)\right) \mathrm{d} y,
\end{array}\right.
$$

where $0 \leq x \leq 1$, the condition $\beta<(1 / 3)$ holds, and $f_{1}(x)$, $f_{2}(x)$, and $f_{3}(x)$ are determined by the exact solution $\left[u_{1}(x) ; u_{2}(x) ; u_{3}(x)\right]=\left[x ; x^{2} ; e^{x}\right]$. The initial vectors $\bar{u}_{p}^{(0)}=0 \quad(p=1,2,3)$ are considered for starting the algorithm. The results for different $n$ are given in Tables 4 and 5. From the computed error results and convergence rates in Tables 4 and 5, we can derive the same performance as Example 1. 
(i) Step 1. Choose $n$ and calculate $x_{i}(i=0,1, \ldots, n)$ along with $h$. Moreover, given the value of $\varepsilon$ and the initial vectors $\bar{u}_{1}^{(0)}=\bar{u}_{2}^{(0)}=0$, set $k=0$.

(ii) Step 2. Solve the nonlinear system $\left\{\begin{array}{l}F_{1 i}=\bar{u}_{1}^{(k+1)}\left(x_{i}\right)-f_{1}\left(x_{i}\right)-\sum_{j=0}^{n} \omega_{j}\left(\lambda_{11} k_{11}^{i j} \phi_{11}\left(x_{j}, \bar{u}_{1}^{(k)}\left(x_{j}\right)\right)+\lambda_{12} k_{12}^{i j} \phi_{12}\left(x_{j}, \bar{u}_{2}^{(k)}\left(x_{j}\right)\right)\right), \\ F_{2 i}=\bar{u}_{2}^{(k+1)}\left(x_{i}\right)-f_{2}\left(x_{i}\right)-\sum_{j=0}^{n} \omega_{j}\left(\lambda_{21} k_{21}^{i j} \phi_{21}\left(x_{j}, \bar{u}_{1}^{(k)}\left(x_{j}\right)\right)+\lambda_{22} k_{22}^{i j} \phi_{22}\left(x_{j}, \bar{u}_{2}^{(k)}\left(x_{j}\right)\right)\right),\end{array}\right.$ where $i=0,1, \ldots, n$ and $k=0,1, \ldots$

(iii) Step 3. In Algorithm 1, using the composite trapezoidal rule $\sum_{j=0}^{n} \lambda_{p q} k_{p q}^{i j} \omega_{j} \phi_{p q}\left(x_{j}, \bar{u}_{q}^{(k)}\left(x_{j}\right)\right)=(h / 2) \sum_{j=0}^{n-1} \lambda_{p q}$ $\left[k_{p q}^{i j} \phi_{p q}\left(x_{j}, \bar{u}_{q}^{(k)}\left(x_{j}\right)\right)+k_{p q}^{i j+1} \phi_{p q}\left(x_{j+1}, \bar{u}_{q}^{(k)}\left(x_{j+1}\right)\right)\right]$ or using the composite Simpson rule $\sum_{j=0}^{n} \lambda_{p q} k_{p q}^{i j} \omega_{j} \phi_{p q}\left(x_{j}, \bar{u}_{q}^{(k)}\left(x_{j}\right)\right)=$ $(h / 6) \sum_{j=0}^{n-1} \lambda_{p q}\left[k_{p q}^{i j} \phi_{p q}\left(x_{j}, \bar{u}_{q}^{(k)}\left(x_{j}\right)\right)+k_{p q}^{i j+1} \phi_{p q}\left(x_{j+1}, \bar{u}_{q}^{(k)}\left(x_{j+1}\right)\right)+4 k_{p q}^{i j+1 / 2} \phi_{p q}\left(x_{j+1 / 2}, \bar{u}_{q}^{(k)}\left(x_{j+1 / 2}\right)\right)\right]$ where $k_{p q}^{i j+1}=k_{p q}\left(x_{i}, x_{j+1}\right)$ and $k_{p q}^{i j+1 / 2}=k_{p q}\left(x_{i}, x_{j+1 / 2}\right), p, q=1,2$.

(iv) Step 4. Calculate the Jacobian matrix $F^{\prime}=\left[\left(\partial F_{1 i} / \partial x_{i}\right) ;\left(\partial F_{2 i} / \partial x_{i}\right)\right]$.

(v) Step 5. Calculate $\left[\bar{u}_{1 i}^{(k+1)} ; \bar{u}_{2 i}^{(k+1)}\right]$ by steps $2-4$.

(vi) Step 6. Compute $\left\{\left\|\bar{u}_{1 i}^{(k+1)}-\bar{u}_{1 i}\right\|_{\infty},\left\|\bar{u}_{2 i}^{(k+1)}-\bar{u}_{2 i}\right\|_{\infty}\right.$. If $\left\{\left\|\bar{u}_{1 i}^{(k+1)}-\bar{u}_{1 i}\right\|_{\infty}<\varepsilon,\left\|\bar{u}_{2 i}^{(k+1)}-\bar{u}_{2 i}\right\|_{\infty}<\varepsilon\right.$, stop; otherwise, set $k=k+1$ and go to step 5 .

Next, we can derive the following convergence result for the iteration method.

Algorithm 1: Iteration algorithm.

TABLE 1: Numerical results for Example 1 by using the composite trapezoidal rule.

\begin{tabular}{lllll}
\hline$n$ & $\left\|u_{1}-\bar{u}_{1}\right\|_{\infty}$ & Rate & $\left\|u_{2}-\bar{u}_{2}\right\|_{\infty}$ & Rate \\
\hline 1 & $6.2974 e-3$ & & $1.4862 e-2$ & \\
2 & $1.3921 e-3$ & 2.1776 & $3.2583 e-3$ & \\
4 & $3.3884 e-4$ & 2.0385 & $7.9146 e-4$ & 2.1894 \\
8 & $8.3829 e-5$ & 2.0151 & $1.9557 e-4$ & 2.0415 \\
16 & $2.0819 e-5$ & 2.0095 & $4.8422 e-5$ & 2.0168 \\
\hline
\end{tabular}

TABLE 2: Numerical results for Example 1 by using the composite Simpson rule.

\begin{tabular}{lllll}
\hline$n$ & $\left\|u_{1}-\bar{u}_{1}\right\|_{\infty}$ & Rate & $\left\|u_{2}-\bar{u}_{2}\right\|_{\infty}$ & Rate \\
\hline 1 & $2.5422 e-4$ & & $6.4248 e-4$ & \\
2 & $1.2619 e-5$ & 4.3324 & $3.1949 e-5$ & \\
4 & $7.0189 e-7$ & 4.1682 & $1.7816 e-6$ & 4.3298 \\
8 & $3.8452 e-8$ & 4.1901 & $9.7781 e-8$ & 4.1645 \\
16 & $2.2774 e-9$ & 4.0776 & $5.8021 e-9$ & 4.1875 \\
\hline
\end{tabular}

TABLE 3: Error estimates for Example 1 by using the methods of $[17,18]$.

\begin{tabular}{lcr}
\hline$n$ & Method of [17] & $\begin{array}{c}\text { Method of [18] } \\
\left\|u_{1}-\bar{u}_{1}\right\|_{\infty}\left\|u_{2}-\bar{u}_{2}\right\|_{\infty}\end{array}$ \\
\hline 1 & $\left\|u_{1}-\bar{u}_{1}\right\|_{\infty}\left\|u_{2}-\bar{u}_{2}\right\|_{\infty}$ & $1.3358 e-24.3043 e-2$ \\
2 & $3.5236 e-29.7588 e-2$ & $7.8067 e-31.1256 e-2$ \\
4 & $9.4328 e-32.7216 e-2$ & $4.5024 e-46.6112 e-4$ \\
8 & $2.4223 e-37.0378 e-3$ & $3.6592 e-54.7854 e-5$ \\
16 & $6.2637 e-41.9103 e-3$ & $3.4139 e-55.0901 e-5$ \\
\hline
\end{tabular}


TABLE 4: Numerical results for Example 2 by using the composite trapezoidal rule.

\begin{tabular}{lllllr}
\hline$n$ & $\left\|u_{1}-\bar{u}_{1}\right\|_{\infty}$ & Rate & $\left\|u_{2}-\bar{u}_{2}\right\|_{\infty}$ & Rate & $\left\|u_{3}-\bar{u}_{3}\right\|_{\infty}$ \\
\hline 1 & $1.7357 e-2$ & & $1.8882 e-2$ & & $4.2653 e-2$ \\
2 & $4.5935 e-3$ & 1.9178 & $5.1775 e-3$ & 1.8667 & $1.0519 e-2$ \\
4 & $1.1051 e-3$ & 2.0554 & $1.2734 e-3$ & 2.0235 & $2.6167 e-3$ \\
8 & $2.7036 e-4$ & 2.0312 & $3.1476 e-4$ & 2.0164 & $6.5183 e-4$ \\
16 & $6.1391 e-5$ & 2.1388 & $7.5873 e-5$ & 2.0526 & $1.6041 e-4$ \\
\hline
\end{tabular}

TABLE 5: Numerical results for Example 2 by using the composite Simpson rule.

\begin{tabular}{lccccc}
\hline$n$ & $\left\|u_{1}-\bar{u}_{1}\right\|_{\infty}$ & Rate & $\left\|u_{2}-\bar{u}_{2}\right\|_{\infty}$ & Rate & $\left\|u_{3}-\bar{u}_{3}\right\|_{\infty}$ \\
\hline 1 & $1.4973 e-4$ & & $4.9234 e-4$ & & $8.3659 e-4$ \\
2 & $9.5114 e-6$ & 3.9765 & $3.1388 e-5$ & 3.9772 & $5.8626 e-5$ \\
4 & $6.0999 e-7$ & 3.9628 & $2.0234 e-6$ & 3.9554 & $3.9241 e-6$ \\
8 & $3.9161 e-8$ & 3.9613 & $1.2866 e-7$ & 3.9751 & $2.5498 e-7$ \\
16 & $2.4815 e-9$ & 3.9801 & $8.1007 e-9$ & 3.9894 & $1.6457 e-8$ \\
\hline
\end{tabular}

\section{Conclusions}

The present paper discussed an iteration method for the system of Fredholm integral equations. The method was based on the composite collocation method. Some theorems have been proved for convergence analysis. The efficiency and accuracy of the proposed method are shown in two numerical examples. From Examples 1 and 2, our numerical results are in agreement with the obtained theoretical results. Moreover, our method is not difficult to extend to deal with integro-differential equations.

\section{Data Availability}

The data used to support the findings of this study are available from the corresponding author upon request.

\section{Conflicts of Interest}

The authors declare that they have no conflicts of interest.

\section{Acknowledgments}

This work is supported by the Science and Technology Plan Project of Hunan Province (Grant No. 2016TP1020) and the Doctoral Scientific Research Foundation of Hengyang Normal University (Grant No. 18D12).

\section{References}

[1] Y. Yang, Z. Tang, and Y. Huang, "Numerical solutions for fredholm integral equations of the second kind with weakly singular kernel using spectral collocation method," Applied Mathematics and Computation, vol. 349, pp. 314-324, 2019.

[2] K. Maleknejad, N. Aghazadeh, and M. Rabbani, "Numerical solution of second kind fredholm integral equations system by using a taylor-series expansion method," Applied Mathematics and Computation, vol. 175, no. 2, pp. 1229-1234, 2006.

[3] M. Didgar, A. Vahidi, and J. Biazar, "Application of taylor expansion for fredholm integral equations of the first kind," Journal of Mathematics, vol. 51, pp. 1-14, 2019.
[4] G. Q. Han and J. Wang, "Extrapolation of nystrom solution for two dimensional nonlinear fredholm integral equations," Journal of Computational and Applied Mathematics, vol. 134, pp. 259-268, 2001.

[5] L. Landweber, "An iteration formula for fredholm integral equations of the first kind," American Journal of Mathematics, vol. 73, no. 3, pp. 615-624, 1951.

[6] A. H. Borzabadi and O. S. Fard, "A numerical scheme for a class of nonlinear fredholm integral equations of the second kind," Journal of Computational and Applied Mathematics, vol. 232, no. 2, pp. 449-454, 2009.

[7] G. Atiyeh Mashhadi, R. Ezzati, and T. Allahviranloo, "Numerical solution of two-dimensional nonlinear fuzzy fredholm integral equations via quadrature iterative method," Journal of Intelligent \& Fuzzy Systems, vol. 36, pp. 661-674, 2019.

[8] C. T. Baker, The Numerical Treatment of Integral Equations, Clarendon Press, Oxford, UK, 1977.

[9] R. Kress, Linear Integral Equations, Springer-Verlag, Berlin, Germany, 1989.

[10] J. Strain, "Locally corrected multidimensional quadrature rules for singular functions," SIAM Journal on Scientific Computing, vol. 16, no. 4, pp. 992-1017, 1995.

[11] A. Meier, T. Arens, S. N. Chandler-Wilde, and A. Kirsch, "A nyström method for a class of integral equations on the real line with applications to scattering by diffraction gratings and rough surfaces," Journal of Integral Equations and Applications, vol. 12, no. 3, pp. 281-321, 2000.

[12] C. Allouch, D. Sbibih, and M. Tahrichi, "Superconvergent nyström method for urysohn integral equations," BIT Numerical Mathematics, vol. 57, no. 1, pp. 3-20, 2017.

[13] T. Okayama, "Theoretical analysis of a sinc-nyström method for volterra integro-differential equations and its improvement," Applied Mathematics and Computation, vol. 324, pp. 1-15, 2018.

[14] M. C. De Bonis and C. Laurita, "On the stability of a modified nyström method for mellin convolution equations in weighted spaces," Numerical Algorithms, vol. 79, no. 2, pp. 611-631, 2018.

[15] J. Zhao, Y. Li, and Y. Xu, "Multiderivative extended rungekutta-nyström methods for multi-frequency oscillatory systems," International Journal of Computer Mathematics, vol. 95, no. 1, pp. 231-254, 2018. 
[16] J. Stoer and R. Bulirsch, Introduction to Numerical Analysis, Springer-Verlag, New York, NY, USA, 1980.

[17] S. Kumar and I. H. Sloan, "A new collocation-type method for Hammerstein integral equations," Journal of Mathematics and Computer Science, vol. 48, pp. 123-129, 1987.

[18] H. Laeli Dastjerdi and F. M. Maalek Ghaini, "Numerical solution of volterra-fredholm integral equations by moving least square method and chebyshev polynomials," Applied Mathematical Modelling, vol. 36, no. 7, pp. 3283-3288, 2012. 